\title{
A REMARK ON ALGEBRAIC GROUPS ATTACHED TO HODGE-TATE MODULES
}

\author{
By Shuji Yamagata
}

Let $K$ be a local field of characteristic 0 with the algebraically closed residue field of characteristic $p>0$. We consider a semi-simple Hodge-Tate module $V$ over $K$ with $V_{\boldsymbol{C}}=\boldsymbol{C} \otimes_{\boldsymbol{Q}_{p}} V=V_{\boldsymbol{C}}(0) \oplus V_{\boldsymbol{C}}(1), n_{0}=\operatorname{dim} V_{\boldsymbol{c}}(0) \geqq 1$ and $n_{1}=\operatorname{dim} V_{\boldsymbol{C}}(1) \geqq 1$. Let $H_{V}$ be the algebraic group attached to $V, H_{V}{ }^{\circ}$ be the neutral component of $H_{V}$ and $\mathrm{g}_{V}$ be their Lie algebra.

In [5] Serre has proved that $H_{V}=\boldsymbol{G} \boldsymbol{L}_{V}$ if $n_{0}$ and $n_{1}$ are relatively prime and if $V$ is an absolutely simple $\mathfrak{g}_{V}$-module. He also remarked the possibility of determination of the structure of $H_{V}{ }^{\circ}$ for other cases. For example, in [6] he has proved that all the irreducible components of the root system of $H_{V}{ }^{\circ}$ are of type A, B, C or D and furthermore are of type $\mathrm{A}$ if $V$ is irreducible of odd dimension.

In this paper we prove that all the irreducible components of the root system of $H_{V}{ }^{\circ}$ are of type $\mathrm{A}$ if $n_{0} \neq n_{1}$ and if $V$ is an absolutely simple $\mathfrak{g}_{V}$-module.

\section{$\S 1$. Irreducible components of the root system.}

In this section we use the following notations (cf. [6], §3).

$\boldsymbol{Q}=$ the field of rational numbers.

$E=$ a field of characteristic 0 .

$\boldsymbol{G}_{m}=$ the one-dimensional multiplicative algebraic group over $E$.

$M=$ a connected reductive algebraic group defined over $E$.

$E^{\prime}=$ a finite Galois extension of $E$ over which $M$ splits.

$\Gamma=$ the Galois group of $E^{\prime} / E$.

$C=$ an algebraically closed field containing $E^{\prime}$.

$T=$ a splitting maximal torus of $M_{/ E^{\prime}}$, where $M_{/ E^{\prime}}$ denotes the scalar extension to $E^{\prime}$ of $M$.

$X=$ the character group of $T$.

$Y=$ the group of the one-parameter subgroups of $T$.

$X_{\mathbf{Q}}=\boldsymbol{Q} \otimes X$.

$Y_{\boldsymbol{Q}}=\boldsymbol{Q} \otimes Y$.

$\langle x, y\rangle\left(x \in X_{\boldsymbol{Q}}, y \in Y_{\boldsymbol{Q}}\right)=$ the canonical bilinear form on $X_{\boldsymbol{Q}} \times Y_{\mathbf{Q}}$.

$R=$ the root system of $M_{/ E^{\prime}}$ relative to $T$.

Received November 30, 1984 
$\left(R_{\imath}\right)_{i \in I}=$ the irreducible components of $R$.

$R^{\vee}=$ the dual root system of $R$.

$R_{\imath} \vee=$ the dual root system of $R_{\imath}$.

$W=W(R)=$ the Weyl group of $R$.

$W\left(R_{\imath}\right)=$ the Weyl group of $R_{\imath}$.

$Y_{Q}^{+}=\left\{y \in Y_{Q} \mid\langle\alpha, y\rangle \geqq 0\right.$ for all $\left.\alpha \in R\right\}$.

$Y^{+}=Y \cap Y_{\mathbf{Q}}^{+}$.

$X_{2}=$ the subspace of $X_{Q}$ generated by $R_{\imath}$.

$Y_{\imath}=$ the subspace of $Y_{Q}$ generated by $R_{\imath} \vee$.

For $x \in X_{Q}$ and $y \in Y_{Q}$,

$x_{\imath}=$ the component of $x$ in $X_{\imath}$.

$y_{2}=$ the component of $y$ in $Y_{\imath}$.

$h_{M}=$ a one-parameter subgroup of $M_{/ C}$ defined over $C$.

$V=$ a linear representation of $M$ over $E$ of finite dimension.

$\Omega(V)=$ the weights of $V$.

$\Omega^{+}(V)=$ the highest weights of the irreducible components of $V_{E^{\prime}}=E^{\prime} \otimes_{E} V$.

We assume the followings:

(i) $V$ is a faithful representation of $M$ over $E$;

(ii) any normal algebraic subgroup $N$ of $M$, defined over $E$, such that $N_{/ C}$ contains $\operatorname{Im}\left(h_{M}\right)$ is equal to $M$;

$(*)$

(iii) the action of $\boldsymbol{G}_{m / C}$ over $V_{C}=C \otimes_{E} V$ defined by $h_{\boldsymbol{M}}$ has exactly two weights $a$ and $b$ with $a<b$.

(We identify the character group of $\boldsymbol{G}_{m}$ with the rational integers $\boldsymbol{Z}$ in the natural way.)

We put $r=b-a$.

The following Lemmas 1, 2, 3 and 4, except for Lemma 2(i), follow as the correspondings of [6], $\S 3$ where $a=0$ and $b=1$ (cf. [2], § 3 Proof of Lemma 3.3).

LEMMA 1. There exists uniquely $h_{0} \in Y^{+}$such that $h_{M}$ and $h_{0}$, considered as homomorphisms of $\boldsymbol{G}_{m / C}$ into $M_{/ C}$, are conjugate each other by an inner automorphism of $M_{1 C}$ and we have

$$
\{\langle\omega, h\rangle \mid \omega \in \Omega(V)\}=\{a, b\} \text { for all } h \in \Gamma W h_{0} .
$$

LEMMA 2. If $\alpha \in R, \alpha^{\vee} \in R^{\vee}, \omega \in \Omega(V)$ and $h \in \Gamma W h_{o}$, we have

(i) $\langle\alpha, h\rangle=0, r$ or $-r$, and so $h / r$ is a weight of $R^{\vee}$.

(ii) $\left\langle\omega, \alpha^{\vee}\right\rangle=0,1$ or -1 .

Lemma 3. Let $\omega \in \Omega^{+}(V)$ and $h \in \Gamma h_{0}$. Then there is at most one element $i \in I$ such that $\omega_{i} \neq 0$ and $h_{i} \neq 0$.

LEMMA 4. For all $i \in I$, there exist $\omega \in \Omega^{+}(V)$ and $h \in(1 / r) \Gamma h_{o}$ such that $\omega_{i} \neq 0$ and $h_{i} \neq 0$. All the couples $\left(\omega_{i}, h_{2}\right)$, thus obtained, are minimal couples of height 1 . (Note. A minimal couple means "un couple minuscule".) 
Proof of Lemmas 1, 2, 3 and 4. Lemma 1 follows as [6], Lemma 2 and its remark; Lemma 2(ii) follows as [6], Lemma 4 by part (i); Lemma 3 follows as [6], Lemma 6; Lemma 4 follows as [6], Proposition 7 ; for Lemma 2(i) we apply [6], "Variante" of Lemma 4.

Proposition. If $M$ is semi-simple and $a+b \neq 0$, then all the irreducible components $R_{\imath}$ of the root system $R$ are of type $A$.

Proof. By Lemma 4, all the $R_{\imath}$ are of type A, B, C or D (cf. [6], Corollary 1 of Proposition 7). We assume that for some $i \in I, R_{\imath}$ is of type $\mathrm{B}, \mathrm{C}$ or $\mathrm{D}$. From Lemma 4, there exist $\omega \in \Omega^{+}(V)$ and $h \in(1 / r) \Gamma h_{o}$ such that $\omega_{i} \neq 0$ and $h_{i} \neq 0$ and $\left(\omega_{i}, h_{\imath}\right)$ is a minimal couple of height 1 . By applying $\S 3$ below to the scalar extension of the root system $R_{\imath}$ and the bilinear form $\left\langle x_{\imath}, y_{i}\right\rangle\left(x_{i} \in X_{\imath}, y_{i} \in Y_{i}\right)$ by which $Y_{\imath}$ is identified with the dual of $X_{\imath}$, we have

$$
\left\{\left\langle w \omega_{i}, h_{\imath}\right\rangle \mid w \in W(R)\right\}=\left\{\left\langle w \omega_{i}, h_{\imath}\right\rangle \mid w \in W\left(R_{\imath}\right)\right\}=\{ \pm(1 / 2)\} .
$$

By Lemma $3, \omega_{j}=0$ or $h_{\jmath}=0$ for all $\jmath \in I$ such that $\jmath \neq i$. In either case, $\left\langle w \omega_{\jmath}, h_{\jmath}\right\rangle=0$ for all $\jmath \in I$ such that $\jmath \neq i$. And so,

$$
\langle w \omega, h\rangle=\sum_{j \in I}\left\langle w \omega_{j}, h_{\jmath}\right\rangle=\left\langle w \omega_{i}, h_{\imath}\right\rangle \quad \text { for all } w \in W(R) .
$$

Thus we have

$$
\left\{\left\langle w \omega, h^{\prime}\right\rangle \mid w \in W(R)\right\}=\{ \pm(r / 2)\}, \quad \text { where } \quad h^{\prime}=r h \in \Gamma h_{0} .
$$

On the other hand, by lemma 1 , we have

$$
\left\{\left\langle w \omega, h^{\prime}\right\rangle \mid w \in W(R)\right\} \subset\{a, b\} .
$$

Hence we have $a=-(r / 2)$ and $b=r / 2$, and so $a+b=0$. This gives a contradiction.

\section{$\S 2$. Hodge-Tate modules with weights 0 and 1 .}

In this section we use the following notations.

$\boldsymbol{Q}_{p}=$ the field of $p$-adic numbers.

$Z_{p}=$ the ring of $p$-adic integers.

$\boldsymbol{Z}_{p}{ }^{\times}=$the group of units of $\boldsymbol{Z}_{p}$.

$K=$ a local field of characteristic 0 with the algebraically closed residue field of characteristic $p>0$. (K is an extension of $\boldsymbol{Q}_{p}$.)

$\bar{K}=$ an algebraic closure of $K$.

$C=$ the completion of $\bar{K}$.

$G=$ the Galois group of $\bar{K} / K$.

$\chi=$ a character of $G$ with infinite image in $\boldsymbol{Z}_{p}{ }^{*}$.

$\boldsymbol{G}_{m}=$ the one-dimensional multiplicative algebraic group over $\boldsymbol{Q}_{p}$.

(Compare with $\boldsymbol{G}_{m}$ in $\S 1$.) 
A Galois module $V$ over $K$ is a $\boldsymbol{Q}_{p}$-space of finite dimension on which $G$ operates continuously. Let $\rho_{V}$ be the homomorphism of $G$ into the vector space automorphisms Aut $(V)$ of $V$ which gives the action of $G$ on $V$. We put $G_{V}=$ $\operatorname{Im}\left(\rho_{V}\right)$.

The action of $G$ on $V$ is extended to the $C$-space $V_{C}=C \otimes_{\boldsymbol{Q}_{p}} V$ by the formula

$$
s\left(\Sigma c_{i} \otimes x_{i}\right)=\Sigma s\left(c_{\imath}\right) \otimes \rho_{V}(s)\left(x_{\imath}\right) \quad\left(s \in G, c_{i} \in C, x_{i} \in V\right) .
$$

Let $g_{V}$ be the Lie algebra of $G_{V}$ (cf. Lemma 6(i) below).

Let $\boldsymbol{G} \boldsymbol{L}_{V}$ be the algebraic group over $\boldsymbol{Q}_{p}$ of the automorphisms of the vector space $V$. Let $H_{V}$ be the smallest algebraic subgroup $H$ of $\boldsymbol{G} \boldsymbol{L}_{V}$ defined over $\boldsymbol{Q}_{p}$ such that $H\left(\boldsymbol{Q}_{p}\right)$ contains $G_{V} . \quad H_{V}{ }^{0}$ denotes the neutral component of $H_{V}$.

In [4], Theorem 4 , Sen defined the canonical operator $\varphi_{V,}$, , with respect to $\chi$, of $V_{C}$ with the above action of $G$. (Sen used the notation $\varphi$.)

For the canonical operator $\varphi_{V,}$, Sen proved

LEMmA 5. ([4], Theorem 11) $\mathrm{g}_{V}$ is the smallest of the $\boldsymbol{Q}_{p}$-subspaces $S$ of $\operatorname{End}_{Q_{p}}(V)$ such that $\varphi_{V, \chi} \in \boldsymbol{C} \otimes_{Q_{p}} S$.

In the rest of this section, we assume

(**) the canonical operator $\varphi_{V, \chi}$ of $V_{C}$ with respect to $\chi$ is semi-simple and its eigenvalues belong to $\boldsymbol{Z}$.

We put

$$
V_{c, \chi}(i)=\left\{x \in V_{c} \mid \varphi_{V, \chi}(x)=i x\right\} \quad \text { for all } \imath \in \boldsymbol{Z} .
$$

By the assumption $(* *)$, we have $V_{\boldsymbol{c}}=\bigoplus_{i \in \boldsymbol{Z}} V_{\boldsymbol{C}, \chi}(i)$. For any $c \in \boldsymbol{G}_{m}(\boldsymbol{C})$, we associate the automorphism $h_{V, \chi}(c)$ defined by the formula

$$
h_{V, x}(c)(x)=c^{2} x \text { for all } i \in \boldsymbol{Z} \text { and all } x \in V_{c, x}(i) \text {. }
$$

Thus we obtain an algebraic group homomorphism $h_{V, \%}$ over $\boldsymbol{C}$ of $\boldsymbol{G}_{m / C}$ into $\boldsymbol{G} \boldsymbol{L}_{V / c}$.

Lemma 6. Let $V$ be as above. Then.

(i) $\mathrm{g}_{V}$ is the Lie algebra of $H_{V}$.

(ii) $H_{V}{ }^{\circ}$ is the smallest algebraic subgroup of $\boldsymbol{G L}_{V}$ defined over $\boldsymbol{Q}_{p}$ whach, after scalar extension to $\boldsymbol{C}$, contains $\operatorname{Im}\left(h_{V}, \chi\right)$.

Proof. (i) follows as [3], Theorem 2 (cf. [6], Theorem 1'). As [6], Theorem 2, (ii) follows from (i) and Lemma 5.

A Galois module $V$ is a Hodge-Tate module if and only if $V$ satisfies the above assumption $(* *)$ with respect to the cyclotomic character $\chi_{o}$, and then $V_{C, \chi_{0}}(i)$ in the above sense coincides with $V_{C}(i)$ in [6], 1.2 (cf. [4], Corollary of Theorem 6). If $V_{\boldsymbol{C}}(i) \neq 0$, we call $\imath$ a weight of the Hodge-Tate module $V$. 
THEOREM. Let $V$ be a Galois module satisfying the assumption (**) above and furthermore $V_{C}=V_{C, x}\left(i_{1}\right) \oplus V_{C, x}\left(i_{2}\right)$ for some $i_{1}, i_{2} \in Z$ with $i_{1}<i_{2}$. We assume that $V$ is an absolutely simple $\mathrm{g}_{V}$-module and that the dimensions $n_{1}$ and $n_{2}$ of $V_{C, \chi}\left(i_{1}\right)$ and $V_{C, \chi}\left(i_{2}\right)$ are different positive integers. Then all the irreducible components of the root system of $H_{V}{ }^{\circ}$ are of type $A$.

Proof. (1) By semi-simplicity of $V, H_{V}{ }^{\circ}$ is reductive. Let $T$ (resp. $S$ ) be the neutral component of the center (resp. the commutator group) of $H_{V}{ }^{\circ}$. Then $T$ and $S$ are defined over $\boldsymbol{Q}_{p}, T \cap S$ is zero-dimensional and we have $H_{V}{ }^{\circ}=T \cdot S$. Also by Lemma $6(\mathrm{i})$ and absolute simplicity of $V, T$ is reduced to $\{1\}$ or equal to the group of homotheties which is identified with $\boldsymbol{G}_{m}$. In either case $S \cap \boldsymbol{G}_{m}$ is zero-dimensional and $S \cdot \boldsymbol{G}_{m}=H_{V}{ }^{\circ} \cdot \boldsymbol{G}_{m}$. Hence we have $\operatorname{dim} S=\operatorname{dim} H_{V}{ }^{\circ} \cdot \boldsymbol{G}_{m}-1$.

(2) We put

$$
n=n_{1}+n_{2}(=\operatorname{dim} V), \quad m=n_{1} i_{1}+n_{2} i_{2} .
$$

Here we have

and

$$
n i_{1}-m \neq n i_{2}-m, \quad\left(n i_{1}-m\right)+\left(n i_{2}-m\right) \neq 0
$$

$$
n_{1}\left(n i_{1}-m\right)+n_{2}\left(n i_{2}-m\right)=0 \text {. }
$$

We remark that it is sufficient to prove this theorem for a finite extension of $K$. After replacing $K$ by a finite extension of $K$, if necessarily, we have a character $\chi^{\prime}$ with infinite image in $\boldsymbol{Z}_{p}{ }^{\times}$such that $\left(\chi^{\prime}\right)^{n}=\chi$ and $\operatorname{Ker} \chi^{\prime}=\operatorname{Ker} \chi$. For the canonical operators $\varphi_{V, \chi^{\prime}}$ and $\varphi_{V, \chi}$ of $V_{C}$ with respect to $\chi^{\prime}$ and $\chi$, we have

$$
n \varphi_{V, \chi}=\varphi_{V, \chi^{\prime}}, \quad V_{c, \chi^{\prime}}\left(n i_{1}\right)=V_{c, \chi}\left(i_{1}\right) \quad \text { and } \quad V_{c, \chi^{\prime}}\left(n i_{2}\right)=V_{c, \chi}\left(i_{2}\right) \text {. }
$$

We put

$$
\rho^{\prime}(s)(x)=\left(\chi^{\prime}\right)^{-m}(s) \rho_{V}(s)(x) \text { for all } s \in G \text { and all } x \in V \text {. }
$$

We obtain a homomorphism $\rho^{\prime}$ of $G$ into Aut $(V)$. We denote $V^{\prime}$ the $\boldsymbol{Q}_{p}$-space $V$ with the action given by $\rho^{\prime}$. Let $\varphi_{V^{\prime}, \chi^{\prime}}$ be the canonical operator of $V^{\prime}{ }_{C}$ with respect to $\chi^{\prime}$. Then we have

and

$$
\varphi_{V^{\prime}, \chi^{\prime}}=\varphi_{V, \chi^{\prime}}-m \cdot i d ., \quad V_{C, \chi^{\prime}}^{\prime}\left(n i_{1}-m\right)=V_{C, \varkappa^{\prime}}\left(n i_{1}\right)
$$

$$
V^{\prime}{ }_{C, z^{\prime}}\left(n i_{2}-m\right)=V_{C, \chi^{\prime}}\left(n i_{2}\right) \text {. }
$$

(id. is the identity on the $C$-space $V^{\prime}{ }_{C}=V_{C}$.)

Especially $\varphi_{V^{\prime}, \chi^{\prime}}$ satisfies the assumption (**) above with respect to $\chi^{\prime}$.

(3) From Lemma 6(ii) and (2), $H_{V^{\prime}}{ }^{\circ}$ is contained in the unimodular group $\boldsymbol{S} \boldsymbol{L}_{V^{\prime}}=\boldsymbol{S} \boldsymbol{L}_{V} . \quad$ By the definitions of $H_{V^{\prime}}$ and $H_{V}, H_{V^{\prime}} \cdot \boldsymbol{G}_{m}$ and $H_{V} \cdot \boldsymbol{G}_{m}$ are both the smallest algebraic subgroup $L$ of $\boldsymbol{G} \boldsymbol{L}_{V^{\prime}}=\boldsymbol{G} \boldsymbol{L}_{V}$ defined over $\boldsymbol{Q}_{p}$ such that $L\left(\boldsymbol{Q}_{p}\right)$ contains $\operatorname{Im}\left(\rho^{\prime}\right) \cdot \boldsymbol{G}_{m}\left(\boldsymbol{Q}_{p}\right)=\operatorname{Im}\left(\rho_{V}\right) \cdot \boldsymbol{G}_{m}\left(\boldsymbol{Q}_{p}\right)$. Hence $H_{V^{\prime}} \cdot \boldsymbol{G}_{m}=H_{V} \cdot \boldsymbol{G}_{m}$ and the neutral component $\left(H_{V^{\prime}} \cdot \boldsymbol{G}_{m}\right)^{\circ}=H_{V^{\prime}}{ }^{\circ} \cdot \boldsymbol{G}_{m}$ of $H_{V^{\prime}} \cdot \boldsymbol{G}_{m}$ coincides with the neutral component 
$\left(H_{\boldsymbol{V}} \cdot \boldsymbol{G}_{m}\right)^{0}=H_{V}^{0} \cdot \boldsymbol{G}_{m}$ of $H_{V} \cdot \boldsymbol{G}_{m}$. Thus we have

$$
\begin{aligned}
S & =\left[H_{V^{0}}, H_{V}{ }^{o}\right]=\left[H_{V}{ }^{0} \cdot \boldsymbol{G}_{m}, H_{V}{ }^{o} \cdot \boldsymbol{G}_{m}\right]=\left[H_{V^{\prime}}{ }^{o} \cdot \boldsymbol{G}_{m}, H_{V^{\prime}}{ }^{o} \cdot \boldsymbol{G}_{m}\right] \\
& =\left[H_{V^{\prime}}, H_{V^{\prime}}{ }^{o}\right] \subset H_{V^{\prime}}{ }^{o} .
\end{aligned}
$$

Because $H_{V^{\prime}}{ }^{\circ} \cap \boldsymbol{G}_{m}$ is zero-dimensional, we have

$$
\operatorname{dim} H_{V^{\prime}}{ }^{\circ}=\operatorname{dim} H_{V^{\prime}}{ }^{\circ} \cdot \boldsymbol{G}_{m}-1=\operatorname{dim} H_{V}{ }^{\circ} \cdot \boldsymbol{G}_{m}-1=\operatorname{dim} S .
$$

Since $H_{V^{\prime}}{ }^{\circ}$ is connected, we have $H_{V^{\prime}}{ }^{\circ}=S$ and so $H_{V^{\prime}}{ }^{\circ}$ is semi-simple.

(4) If we put $E=\boldsymbol{Q}_{p}, C=\boldsymbol{C}, \quad M=H_{V^{\prime}}{ }^{\circ}, \quad V=V^{\prime}, h_{M}=h_{V^{\prime}, \chi^{\prime}}, \quad a=n i_{1}-m$ and $b=n i_{2}-m$, the assumptions $\left(^{*}\right)$ of $\S 1$ are satisfied: (i) is evident, (ii) results from Lemma 6(ii), and (iii) is obtained in (2). Also the hypotheses of Proposition of $\S 1$ are satisfied: $M$ is semi-simple by (3), and $a+b \neq 0$ by (2). Hence the irreducible components of the root system of $H_{V^{\prime}}{ }^{\circ}=S$ (by (3)) are of type A, so all the irreducible components of the root system of $H_{V}{ }^{\circ}$ are of type A.

Remark. In the above proof, absolute simplicity, not semi-simplicity, is needed only to prove $T=\{1\}$ or the group of homotheties (cf. [6], Remark of Proposition 8).

The following Corollary is a special case of the above Theorem.

Corollary. Let $V$ be a Hodge-Tate module with werghts 0 and 1 . Assume that $V$ is an absolutely simple $\mathrm{g}_{V}$-module and that the dimensions of $V_{C}(0)$ and $V_{c}(1)$ are different positive integers. Then all the irreducible components of the root system of $H_{V}{ }^{\circ}$ are of type $A$.

\section{$\S 3$. Tables of minimal couples of height 1 .}

In this section we use the same notations as in [1], Ch. VI Planches.

For each $R$, in the finite dimensional real vector space $V$, of the following reduced irreducible root systems, we identify the dual space $V^{*}$ of $V$ with $V$ by the positive definite symmetric bilinear form $(x \mid y)$ on $V$, which is invariant under the Weyl group $W(R)$ of $R$. By this identification, we have

$$
\langle x, y\rangle=(x \mid y) \text { for all } x \in V \text { and all } y \in V^{*},
$$

where $\langle x, y\rangle$ is the canonical bilinear form on $V \times V^{*}$, and

$$
\alpha^{\vee}=2 \alpha /(\alpha \mid \alpha) \quad \text { for all } \alpha \in R \text {. }
$$

Let $\left\{\alpha_{1}, \cdots, \alpha_{l}\right\}$ be the basis of $R$, numbered as in [1], Ch. VI Planches. Let $\left\{\omega_{1}, \cdots, \omega_{l}\right\}$ be the fundamental weights of $R$ corresponding to $\left\{\alpha_{1}, \cdots, \alpha_{l}\right\}$ and $\left\{\omega_{1}^{\vee}, \cdots, \omega_{l}^{\vee}\right\}$ be the fundamental weights of the dual $R^{\vee}$ of $R$ corresponding to $\left\{\alpha_{1}^{\vee}, \cdots, \alpha_{l}^{\vee} \vee\right.$.

By [1], Ch. VI Planches and [6], Annex, we have:

Type $A_{l}(l \geqq 1)$ 
minimal couples of height 1: $\left(\omega_{1}, \omega_{i}^{\vee}\right),\left(\omega_{l}, \omega_{i}^{\vee}\right),\left(\omega_{i}, \omega_{1}^{\vee}\right)$ and $\left(\omega_{i}, \omega_{l}^{\vee}\right)$ with $1 \leqq i \leqq l$.

$\omega_{i}^{\vee}=\omega_{2}=\varepsilon_{1}+\cdots+\varepsilon_{i}-\frac{i}{l+1} \sum_{j=1}^{l+1} \varepsilon_{j}$.

$W(R) \omega_{i}=\left\{\varepsilon_{\sigma(1)}+\cdots+\varepsilon_{\sigma(i)}-\frac{i}{l+1} \sum_{j=1}^{l+1} \varepsilon_{j} \mid \sigma \in \Im_{l+1}\right\}$,

where $\widetilde{S}_{l+1}$ is the symmetric group of degree $l+1$.

$\left\langle W(R) \omega_{1}, \omega_{i}^{\vee}\right\rangle=\left(W(R) \omega_{1} \mid \omega_{\imath}\right)=\left\{-\frac{i}{l+1}, \frac{l+1-i}{l+1}\right\}$.

$\left\langle W(R) \omega_{l}, \omega_{i}^{\vee}\right\rangle=\left(W(R) \omega_{l} \mid \omega_{i}\right)=\left\{\frac{i}{l+1}, \frac{i-l-1}{l+1}\right\}$.

$\left\langle W(R) \omega_{i}, \omega_{1}^{\vee}\right\rangle=\left(W(R) \omega_{i} \mid \omega_{1}\right)=\left\{-\frac{i}{l+1}, \frac{l+1-i}{l+1}\right\}$.

$\left\langle W(R) \omega_{i}, \omega_{l}^{\vee}\right\rangle=\left(W(R) \omega_{i} \mid \omega_{l}\right)=\left\{\frac{i}{l+1}, \frac{i-l-1}{l+1}\right\}$.

Type $B_{l}(l \geqq 2)$

minimal couple of height 1: $\left(\omega_{l}, \omega_{1}^{\vee}\right)$.

$\omega_{l}=\left(\varepsilon_{1}+\varepsilon_{2}+\cdots+\varepsilon_{l}\right) / 2$.

$\omega_{1} \vee=\omega_{1}=\varepsilon_{1}$.

$W(R) \omega_{l}=\left\{\left( \pm \varepsilon_{1} \pm \varepsilon_{2} \pm \cdots \pm \varepsilon_{l}\right) / 2\right\}$.

$\left\langle W(R) \omega_{l}, \omega_{1}^{\vee}\right\rangle=\left(W(R) \omega_{l} \mid \omega_{1}\right)=\{ \pm(1 / 2)\}$.

Type $C_{l}(l \geqq 2)$

minimal couple of height 1: $\left(\omega_{1}, \omega_{l}^{\vee}\right)$.

$\omega_{1}=\varepsilon_{1}$.

$\omega_{l}^{\vee}=\left(\omega_{l}\right) / 2=\left(\varepsilon_{1}+\varepsilon_{2}+\cdots+\varepsilon_{l}\right) / 2$.

$W(R) \omega_{1}=\left\{ \pm \varepsilon_{1}, \pm \varepsilon_{2}, \cdots, \pm \varepsilon_{l}\right\}$.

$\left\langle W(R) \omega_{1}, \omega_{l}^{\vee}\right\rangle=\left(W(R) \omega_{1} \mid\left(\omega_{l}\right) / 2\right)=\{ \pm(1 / 2)\}$.

Type $D_{l}(l \geqq 4)$

minimal couples of height 1

for $l=4:\left(\omega_{i}, \omega_{j}^{\vee}\right)$ with $i, j \in\{1,3,4\}$ and $i \neq j$

for $l \geqq 5$ : $\left(\omega_{1}, \omega_{l-1}^{\vee}\right),\left(\omega_{1}, \omega_{l}^{\vee}\right),\left(\omega_{l-1}, \omega_{1}^{\vee}\right)$ and $\left(\omega_{l}, \omega_{1}^{\vee}\right)$.

$\omega_{1}^{\vee}=\omega_{1}=\varepsilon_{1}$. 
$\omega_{l-1} \vee=\omega_{l-1}=\left(\varepsilon_{1}+\varepsilon_{2}+\cdots+\varepsilon_{l-1}-\varepsilon_{l}\right) / 2$.

$\omega_{l}^{\vee}=\omega_{l}=\left(\varepsilon_{1}+\varepsilon_{2}+\cdots+\varepsilon_{l-1}+\varepsilon_{l}\right) / 2$.

$W(R) \omega_{1}=\left\{ \pm \varepsilon_{1}, \pm \varepsilon_{2}, \cdots, \pm \varepsilon_{l}\right\}$.

$W(R) \omega_{l-1}=\left\{\left(\xi_{1} \varepsilon_{1}+\xi_{2} \varepsilon_{2}+\cdots+\xi_{l} \varepsilon_{l}\right) / 2 \mid \xi_{\imath}= \pm 1, \prod_{\imath} \xi_{\imath}=-1\right\}$.

$W(R) \omega_{l}=\left\{\left(\xi_{1} \varepsilon_{1}+\xi_{2} \varepsilon_{2}+\cdots+\xi_{l} \varepsilon_{l}\right) / 2 \mid \xi_{\imath}= \pm 1, \prod_{\imath} \xi_{\imath}=1\right\}$.

$\left\langle W(R) \omega_{i}, \omega_{j}^{\vee}\right\rangle=\left(W(R) \omega_{i} \mid \omega_{j}\right)=\{ \pm(1 / 2)\}$ for all $(i, j)$ as above.

\section{REFERENCES}

[1] N. Bourbaki, Éléments de Mathématique, Groupes et algèbres de Lie, chapitres IV, V et VI, Hermann, 1968.

[2] F. HAzAma, Algebraic cycles on certain abelian varieties and powers of special surfaces, J. Fac. Sci. Univ. Tokyo Sect. IA, Math. 31 (1984), 487-520.

[3] S. SEN, Lie algebras of Galois groups arising from Hodge-Tate modules, Ann. of Math. 97 (1973), 160-170.

[4] S. SEN, Continuous cohomology and $p$-adic Galois representations, Inventiones math. 62 (1980), 89-116.

[5] J-P. SERRE, Sur les groupes de Galois attachés aux groupes $p$-divisibles, Proc. Conf. Local Fields (T. A. Springer edit.), Springer-Verlag, Heidelberg, 1967, 118131.

[6] J-P. Serre, Groupes algébriques associés aux modules de Hodge-Tate, Astérisques 65, Soc. Math. de France (1979), 155-188.

Department of Mathematical Science

College of Science and Engineering

TOKYO DENKI UNIVERSITY

HATOYAMA-MACHI, HiKI-GUN

SAITAMA-KEN, JAPAN 